\title{
Research of subway tunnel staff positioning system based on UWB technology
}

\author{
Mingdong Wang \& Yawen Dai \\ Wuhan University of Technology, Wuhan, China.
}

\begin{abstract}
Aiming at the problems of short transmission distance, low multipath effect and low positioning accuracy in the common tunnel personnel positioning technology, proposed a new system of personnel positioning system for subway tunnels based on the Impulse UWB (Impulse Radio-Ultra Wideband, IRUWB). According to the characteristics of the tunnel complex environment, the system can reasonably utilize the advantages of the time difference of arrival (TDOA) and the time of arrival (TOA) to achieve a reasonable position in different parts of the whole tunnel. The whole system is to send the ranging signal to the positioning sensor through the tunnel staff who wear electronic tags, and the use of Symmetric Double-Sided TwoWay Ranging to calculate the exact position of personnel information.
\end{abstract}

KEYWORD: Personnel positioning; Impulse Radio-Ultra Wideband; TDOA; TOA; SDS-TWR

\section{INTRODUCTION}

With the traffic jam problem of large and medium cities in the country increasingly grim. Subway because of its large capacity, small pollution and other advantages, has become the first choice of major cities to ease congestion. But in the construction of subway tunnel, due to the existence of closed strong, multi-structure, communications inconvenience, harsh environment and other characteristics, caused a serious threat to the construction personnel of the personal safety. Therefore, in addition to taking security measures, but also the need for timely rescue measures. The precise location of the subway tunnel personnel is one of the most important information to rescue the trapped workers.

However, most of positioning technologies have some problems, such as short transmission distance, poor anti multipath effect, low positioning accuracy. Aiming at these problems, proposed a mine personnel positioning system based on UWB, Make full use of UWB technology has a strong anti-jamming performance, high transfer rate, low power consumption, small transmission power and other characteristics, and the TDOA and TOA location algorithm to obtain real-time precise positioning tunnel officer. The experimental results show that the method of tunnel personnel positioning has good stability and high positioning accuracy.

\section{ULTRA-WIDEBAND PRINCIPLE}

\subsection{Ultra-wideband presentation}

Ultra wide band technology is the use of very narrow pulse signal to achieve wireless communication, called pulse radio. In 1989, the US Defense Advanced Research Projects Agency (DARPA) first used the term UWB. February 2002, UWB technology was first approved by the US Federal Communications Commission for civilian communications, Approval of the $3.1-10.6 \mathrm{GHz}$ free authorization band assigned to the use of UWB, and released a commercial definition of UWB technology:

First, the relative bandwidth of the signal is not less than 0.2 , namely

$B_{f}=\frac{2\left(f_{H}-f_{L}\right)}{f_{H}+f_{L}} \geq 0.2$

In the formula, $\mathrm{B}_{\mathrm{f}}$ is the relative signal bandwidth; $\mathrm{f}_{\mathrm{H}}$ is the highest frequency of the signal; $f_{L}$ is the lowest frequency of the signal.

Second, the bandwidth of the signal is absolutely no less than $500 \mathrm{MHz}, 10 \mathrm{~dB}$ bandwidth refers to the bandwidth that is

$B_{a}=\left(f_{H}-f_{L}\right) \geq 500 M H z$

In the formula, $\mathrm{B}_{\mathrm{a}}$ is the absolute bandwidth of the signal. 
The distance resolution of the radio signal is directly related to the bandwidth of the signal. Due to the broadband characteristics of the signal, the range resolution accuracy of UWB system is much higher than that of narrow band wireless system. Using a pulse width of nanosecond or sub-nanosecond, theoretically possible to obtain the ranging accuracy of centimeter or even millimeter levels. UWB emission is extremely short duration and duty ratio is very low and narrow pulse, so the multipath signal in time is separable. In addition, UWB uses spreadspectrum signal, the system has a wider frequency band. According to the Shannon formula, the channel capacity $\mathrm{C}$ under certain circumstances, high bandwidth can reduce the signal to noise ratio, therefore, UWB has a strong anti-interference.

\subsection{Signal modulation}

In this paper, the IR-UWB is a Gaussian pulse signal, the time-domain waveform expression is as follows:

$$
p(t)= \pm \frac{1}{\sqrt{2 \pi \sigma^{2}}} e^{-\left(\frac{r^{2}}{2}\right)}= \pm \frac{\sqrt{2}}{a} e^{\frac{2 \pi t^{2}}{\alpha^{2}}}
$$

Here, $\alpha^{2}=4 \pi \sigma^{2}$ is the pulse forming factor, $\sigma^{2}$ is the variance. In order to ensure the effective radiation of the pulse energy, the pulse should not contain DC component, Gauss function of the signal waveform can be shown to meet this condition. The actual signal waveform is usually the two - order Gauss function. In this way, we can change the pulse width, the differential of the pulse and the combination of the base function to change the spectrum structure of the impulse pulse, which can meet the requirements of the power limit and frequency spectrum of FCC. In this paper, the development of high-precision radio positioning system is based on the IEEE802.15.42011 standard IR-UWB technology. Using DW1000 chip of Decawave company design of radio frequency positioning system communication node, the application of SDS-TWR to achieve ranging. The maximum bandwidth of the signal is $1300 \mathrm{MHz}$.

\section{SIGNAL TWR-TOF RANGING PRINCIPLE}

\subsection{Ranging Analysis}

Wireless positioning system to realize the orientation, generally the first goal is obtain relevant measurement parameters of target position and establish corresponding mathematical model, and then use these parameters and the establishment of the mathematical model to calculate the position coordinates of the target. And the most important measurement parameter in the whole positioning system is the precision of Flight of Time (TOF). According to the calculation formula of the Cramer-Rao Bound, the accuracy is proportional to the signal bandwidth, namely IR-UWB signals with high temporal resolution. Therefore, compared with the traditional narrow band signal can obtain a higher time measurement accuracy. Secondly the measurement accuracy based on TOF positioning technology is very easy to be affected by multipath interference in practical applications. While the IR-UWB signal is used second order Gaussian pulse in the time domain, usually $0.1 \sim 0.5 \mathrm{~ns}$. This makes it easier to separate the multipath reflection signal and the direct signal on the time axis in the multipath environment. That compared to a narrowband signal having a better multipath resolution capability. Finally, the signal time of flight ranging system clock jitter on TOF can not be ignored. 1ns time measurement errors can also cause the error distance of $30 \mathrm{~cm}$. The crystal drift caused by the clock jitter is inevitable. For IR-UWB signal has these advantages and oscillator drift error, proposes a scheme based on SDS-TWR location algorithm.

\subsection{SDS-TWR Ranging principle}

SDS-TWR ranging process as shown in Figure 1.At the beginning of the range, first, the tag sends a poll signal that contains the ID and marks the time stamp Tsp.The anchor node receives the poll signal and marks the poll arrival time stamp $\mathrm{T}_{\mathrm{RP}}$. Because there is no clock synchronization mechanism between Tag and Anchor, ranging transmission time $T_{p}$ can not be obtained directly. And after bilateral ranging, A response signal is given to the tag after the anchor node is delayed TRSP. The tag receives the response signal and marks the time of arrival $T_{R R}$ of the signal. Thus it can be obtained:

$$
2 T_{p}=\left(T_{R R}-T_{S P}\right)-\left(T_{S R}-T_{R P}\right)
$$

Consider the impact of the system oscillator drift, then the actual measurement results should be

$$
2 \hat{T}_{p}=\left(T_{R R}-T_{S P}\right)\left(1+e_{T}\right)-\left(T_{S R}-T_{R P}\right)\left(1+e_{A}\right)
$$

Tyре: $\mathrm{e}_{\mathrm{T}}, \mathrm{e}_{\mathrm{A}}$ respectively for crystal oscillator drift measurement error of label and anchor node

The $T_{R R}-T_{S P}=2 T_{p}+T_{R S P}$ and $T_{S R}-T_{R P}=T_{R S P}$ are substituted into the formula (5) obtained

$$
\hat{T}_{p}-T_{p}=\frac{1}{2}\left(T_{R S P} \times e_{T}-T_{R S P} \times e_{A}+2 T_{p} \times e_{T}\right)
$$

As the $T_{\text {RSP }}$ is much larger than $T_{p}$, Then the above equation simplifies to

$$
\hat{T}_{p}-T_{p}=\frac{1}{2} T_{R S P} \times\left(e_{T}-e_{A}\right)
$$

By equation (7) can be drawn TWR by crystal oscillator drift error $\mathrm{e}_{\mathrm{T}}$ and $\mathrm{e}_{\mathrm{A}}$ of the differential operation, reduce the influence of crystal oscillator 
drift, so as to reduce the measurement error. To further improve the ranging accuracy can be used SDSTWR on this basis. As shown in figure 1, After the completion of TWR, The tag receives the Response signal from the anchor node, TRSP delay time, Tag sends a Final signal, After the anchor node receives Final Signal, The two bilateral ranging completed, Is SDS-TWR ranging. The formula for calculating of the ranging result is:

$$
4 T_{p}=\left(T_{R R}-T_{S P}\right)-T_{S R P}+\left(T_{R F}-T_{S R}\right)-T_{S R P}
$$

Also consider oscillator drift, in accordance with formula (7)and the formula (8) can be obtained

$\hat{T}_{p}-T_{p}=\frac{1}{4} \times\left(T_{R S P t}-T_{R S P a}\right) \times\left(e_{T}-e_{A}\right)$

Since $\mathrm{T}_{\mathrm{RSP}}$ is much larger than $\mathrm{TRSP}_{\mathrm{R}}-\mathrm{T}_{\mathrm{RSP}}$, namely contrast of formula (7) and (9), SDS-TWR algorithm can be obtained with respect to the higher range accuracy than TWR algorithm. This can be in the premise of without the use of high-precision crystal premise, to obtain a higher accuracy of TOF.

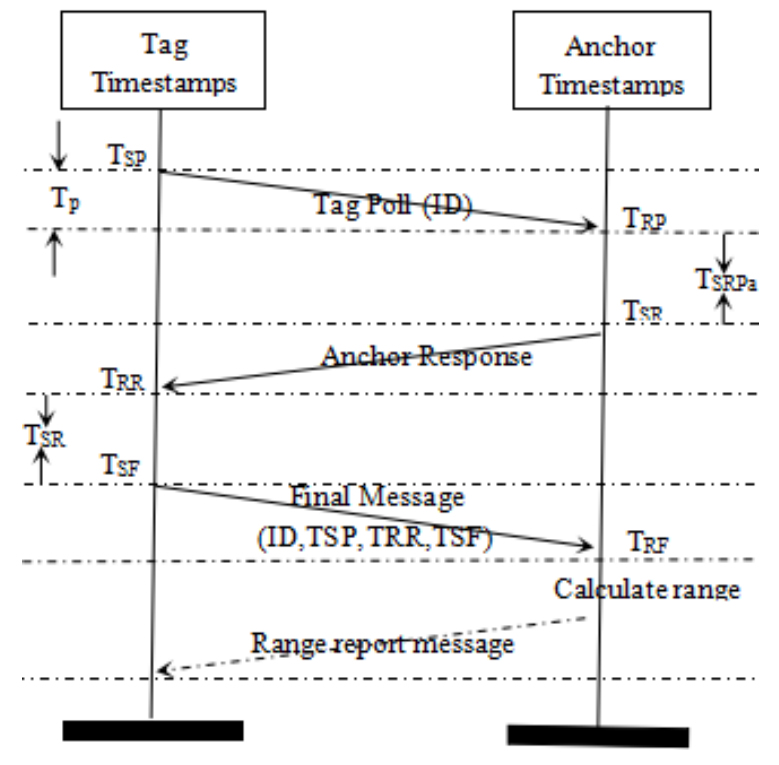

Figure 1. Schematic diagram of SDS-TWR distance measurement

\section{ULTRA-WIDEBAND LOCATION ALGORITHM}

\subsection{Introduction of two positioning algorithms}

Common UWB positioning technology based on Time of Arrival(TOA) positioning algorithm or Time Difference of Arrival(TDOA) positioning algorithm. Based on TDOA location algorithm uses ranging procedure, the linear distance between the position sensor and the destination node. According to the results of each TOA distance measurement get a circle corresponding to the equation. And based on the positioning of the TDOA is also using the dis- tance measurement method, but the distance difference between the 2 positioning sensors and the target node is obtained. Then a corresponding hyperbolic equation is obtained from the result of each TDOA distance measure.

\subsection{Comparison of two kinds of localization algorithms}

The biggest advantage of UWB positioning technology based on TOA is to avoid the clock synchronization. On the one hand, it greatly reduces the complexity of the system, Without considering the complex synchronization solutions, and the impact of synchronization errors ranging positioning; On the other hand, as long as a reasonable positioning sensors designed access mechanisms to effectively prevent mutual interference between the positioning sensor can accurately measure the propagation time of the pulse signal. However, UWB based TOA positioning technology can support the positioning of the number of personnel is limited, and the positioning of the accuracy is limited. UWB positioning technology based on TDOA is not the direct use of signal arrival time, but the use of a plurality of positioning sensor receives the signal arrival time difference to determine the coordinate position of the target node. Compared with the UWB positioning technology based on TOA, based on TDOA location technology of UWB although the need for clock synchronization, but the positioning accuracy has been greatly improved, it can support multi-user real-time high-precision positioning.

\subsection{The realization of the system positioning algorithm}

According to the advantages of these two kinds of positioning technology and the environmental characteristics of the subway tunnel, We will be appropriate to take the appropriate positioning technology, Namely in the subway tunnel construction, the staff is more intensive, and the space is relatively small, the need for multi-user real-time high-precision positioning, Adoption of UWB based on TDOA location technology to achieve the two-dimensional spatial coordinates of high-precision positioning is the best option; And in the tunnel has been completed, because of its personnel are more sparse, and the space is relatively narrow and long, only the user can be roughly accurate positioning. That's UWB based TOA positioning technology to achieve precise spatial positioning of one-dimensional coordinate system to meet the parts of the tunnel have been completed targeting requirements. This allows personnel positioning system constructed in different areas throughout the subway tunnel.

Using UWB based TDOA positioning technology, the positioning principle diagram shown in Figure 2 
(left). Assume that the position of the moving tag coordinates is $(\mathrm{x}, \mathrm{y})$, The position coordinate of the position sensor is $\left(x_{i}, y_{i}\right), i=1,2,3,4$. From TDOA ranging mechanism, Clock synchronization is required between the positioning sensors, the clock deviation of the positioning sensor and the target node is the same. Suppose this deviation is $\Delta \mathrm{t}$, The spatial geometric relationship between the anchor node and the mobile tag is as follows:

$\sqrt{\left(x-x_{i}\right)^{2}+\left(y-y_{i}\right)^{2}}=c\left(\tau_{i}-\Delta t\right)$

In the formula, $\mathrm{C}$ is the transmission speed of electromagnetic wave in air, $\tau_{\mathrm{i}}$ is the TOF of the signal between the sensor and the target node $\mathrm{i}$

The elimination of clock offset in the formula (10).In this way, we can obtain the position information $\tau_{\mathrm{i}}$ of the mobile tags by using the 4 positioning sensors, And combined with the solution of the equation group, the coordinates of the mobile tags are derived.

Using UWB based TOA positioning technology, the positioning principle diagram is shown in Figure 2 (right). Transmitting a ranging signal from the mobile tab $(\mathrm{x}, \mathrm{y})$ to the positioning sensor ( $\mathrm{xi}_{\mathrm{i}}$, $\mathrm{y}_{\mathrm{i}}$ ), By the distance between the mobile label and two positioning sensors, it can directly determine the location of the mobile tag.

UWB technology based on the subway tunnel personnel positioning system components: Mobile tags, positioning sensors and positioning server, as shown in Figure 2. All positioning sensor locations are determined in advance, and the mobile tag is carried by the tunnel personnel to determine its location.

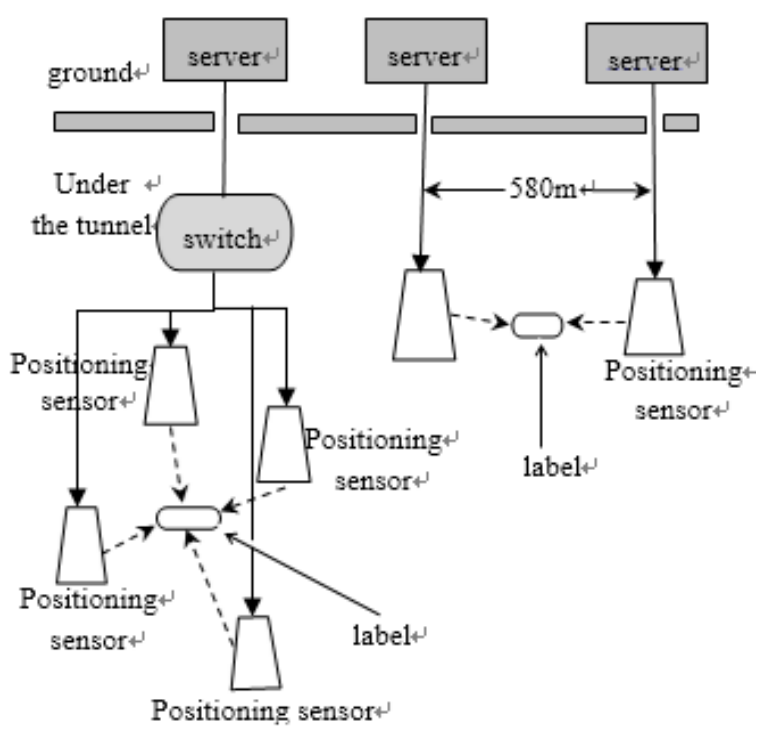

Figure 2 the composition of subway tunnel personnel positioning system based on IR-UWB Technology

\section{CONCLUSION}

The camera-ready copy of the complete paper print Subway tunnel officer position is secure tunnel staff to ensure not only a reasonable allocation of staff under the tunnel, but also in the event of mine accidents, timely and accurately locate trapped people and rescue them.

Positioning system based on IR-UWB technology is the best physical layer technology for high precision indoor positioning at present. By testing the accuracy of ranging and positioning in the room. Experimental results show that, IR-UWB positioning system with strong anti-jamming performance, high transfer rate, low power consumption, transmission power and other characteristics, can achieve decimeter positioning accuracy. The field-proven through subway tunnels, the construction of the tunnel, based TDOA location algorithm, can real-time personnelintensive high-precision positioning. And in the long and narrow tunnel has been completed, the use of TOA based positioning algorithm, can achieve the accurate positioning of personnel. Therefore, it shows that the tunnel personnel positioning system based on IR-UWB has high precision and good reliability in the whole tunnel positioning. Meet the requirements of the personnel in the subway tunnel.

\section{REFERENCES}

Chi Hongpeng. 2012. Automatic guidance of underground mining vehicles using laser sensors. Tunnelling and Underground Space Technology.

CHEHRI A. 2009. UWB based sensor net-works for localization in mining environments. Ad Hoc Net-works.

GEZICI S\&POOR H. 2009. Position estimation via ultra wide band signals. Proceedings of the IEEE.

Li Hui. 2013. Ultra-wideband pulse Communication Technology. Information and Communication.

Wang Fangqiu. 2014. Root-MUSIC-based joint TOA and DOA estimation in IR-UWB. Journal on Communications.

Zheng Lingwen\&Tan zhenhui. 2007. New TDOA algorithm based on Taylor series expansion in cellular networks. Journal on Communications. 